\title{
Kawasaki disease shock syndrome: a rare and severe complication of Kawasaki disease
}

\author{
Mustafa Çakan¹, Hakan Gemici², Nuray Aktay-Ayaz¹, Gonca Keskindemirci², Helen Bornaun², \\ Tarkan İkizoğlu ${ }^{4}$, Alpay Çeliker ${ }^{5}$ \\ Clinics of ${ }^{1}$ Pediatric Rheumatology, ${ }^{2}$ Pediatrics, and ${ }^{3}$ Pediatric Cardiology, Kanuni Sultan Süleyman Research and Training \\ Hospital, ${ }^{4}$ Clinic of Pediatrics, Acıbadem Maslak Hospital and ${ }^{5}$ Division of Pediatric Cardiology, Department of Pediatrics, \\ Koç University Faculty of Medicine, Istanbul, Turkey. \\ E-mail: mustafacakan@hotmail.com
}

Received: 8th September 2015, Revised:6th October 2015, Accepted: 2nd December 2015

\begin{abstract}
SUMMARY: Çakan M, Gemici H, Aktay-Ayaz N, Keskindemirci G, Bornaun H, İkizoğlu T, Çeliker A. Kawasaki disease shock syndrome: A rare and severe complication of Kawasaki disease. Turk J Pediatr 2016; 58: 415-418.

Kawasaki disease is an acute systemic vasculitis that occurs most commonly in young children. It affects medium-sized muscular arteries and the coronary arteries are the predominant site of involvement. Morbidity and mortality is generally due to coronary artery aneurysms that develop during the chronic phase. Although it is well known that Kawasaki disease can cause myocarditis, tachycardia and heart failure during acute stage, Kawasaki disease shock syndrome has been recently described. It is characterized by hypotension, signs and symptoms of poor perfusion and a shock-like state. Herein we describe two cases of Kawasaki disease shock syndrome that were treated in the pediatric intensive care unit and followed a course without morbidity or mortality.
\end{abstract}

Key words: child, intensive care unit, Kawasaki disease, Kawasaki disease shock syndrome, toxic shock syndrome.

Kawasaki disease (KD) is an acute selflimited systemic vasculitis that affects medium-sized arteries ${ }^{1}$. Diagnosis is based on clinical features that are; fever for more than five days accompanied by bilateral conjunctivitis, oropharyngeal changes, cervical lymphadenopathy, polymorphous rash, and peripheral extremity changes 1,2 . Coronary artery aneurysms or ectasia develop in $15 \%$ to $25 \%$ of untreated children and may lead to myocardial infarction, sudden death, or ischemic heart disease $^{2}$. KD shock syndrome (KDSS) is defined as hemodynamic instability during the acute phase of the disease. The cause of KDSS is unknown as $\mathrm{KD}$, but capillary leakage due to vasculitis, myocardial dysfunction, and cytokine dysregulation are thought to be responsible. The patients are hypotensive and show signs and symptoms of poor perfusion ${ }^{3}$. This report describes two children with KDSS, the first one had the diagnosis of KD but the second one initially had the diagnosis of toxic shock syndrome.

\section{Case Reports}

Case 1

An 11-year-old boy was referred to our hospital with the provisional diagnosis of KD. He had high spiking and remittent fever with peak temperatures $>39.6{ }^{\circ} \mathrm{C}$ for 6 days and one dose of intravenous immunoglobulin (IVIG) (2 $\mathrm{g} / \mathrm{kg}$ ) was given on the 5 th day of fever and acetylsalicylic acid was initiated. On physical examination, he had all the signs of complete KD namely; bilateral conjunctivitis, left cervical lymphadenopathy $(2 \times 2 \mathrm{~cm})$, macular rash on the trunks and extremities, edema on the hands and oropharyngeal hyperemia. He had lethargy and drowsiness with fever and severe myalgia that was aggravated by movement and palpation. On laboratory tests leukocyte count was $16,100 / \mathrm{mm}^{3}$, hemoglobin was $9.7 \mathrm{~g} / \mathrm{dl}$, thrombocyte count was 219,000/ $\mathrm{mm}^{3}$, C-reactive protein (CRP) was $369 \mathrm{mg} / \mathrm{L}$ (normal: < 5), erythrocyte sedimentation rate (ESR) was $99 \mathrm{~mm} / \mathrm{hr}$. Liver and kidney function 
tests, muscle enzymes, and urinalysis were within normal limits. Work-up for infection did not yield any possible causative agent (Table I). A second dose of IVIG (2 g/kg) was given by 12 hours of infusion on the same day of admission as fever persisted after 36 hours of the first IVIG infusion. On the 2nd day of hospitalization, he was subfebrile but developed tachycardia, hypotension $(80 / 50 \mathrm{mmHg})$, oliguria, mild dyspnea, and cold extremities. The patient was transferred to the pediatric intensive care unit (PICU). Echocardiography revealed first-degree mitral valve regurgitation with dilatation of the left anterior descending (4.4 mm, z-score:5.4) and right $(3.6 \mathrm{~mm}, \mathrm{z}$-score:2.7) coronary arteries, and ejection fraction (EF) was 50\%. On ultrasound examinations gall bladder was hydropic and there were bilateral $20 \mathrm{~mm}$ pleural effusions. Blood albumin level was $2.1 \mathrm{~g} / \mathrm{dl}$, pro-brain natriuretic peptide (pro-BNP) was 35,000 pg/ml (normal: < 65), troponin-T was $0.127 \mathrm{ng} / \mathrm{ml}$ (normal: <0.014), and INR was mildly prolonged. Fluid resuscitation, broadspectrum antibiotics and milrinone treatment were commenced. Albumin and fresh frozen plasma (FFP) infusions were given. As his fever did not subside, high-dose methylprednisolone (30 mg/kg/day) was given for 3 days. The fever subsided after the second dose and the clinical picture of the patient started to improve on the 5th day. The patient developed typical thrombocytosis (platelet: $707,000 / \mathrm{mm}^{3}$ ) and periungual desquamation of the $\mathrm{KD}$ on the 14th day. Echocardiographic examination was normal on the 4th week without coronary abnormalities. The patient has been followed for 12 months without any complications.

\section{Case 2}

An 8-year-old girl was admitted to the infectious diseases ward with complaints of fever for three days and rash for one day. On physical examination, she had fever $\left(39.5^{\circ} \mathrm{C}\right)$, tachycardia, hepatomegaly, and macular rash on the extremities that was resembling scarlet fever. She was very lethargic, drowsy and also had severe myalgia and weakness that was causing the child to be bedridden. On laboratory tests leukocyte count was 7,700/ $\mathrm{mm}^{3}$, hemoglobin concentration was $11.8 \mathrm{~g} /$ $\mathrm{dl}$, thrombocyte count was $185,000 / \mathrm{mm}^{3}$, CRP was $140 \mathrm{mg} / \mathrm{L}$, and ESR was $30 \mathrm{~mm} / \mathrm{hr}$. She had mild hypertransaminasemia (AST:62
U/L, ALT:99 U/L), and albumin was $3.1 \mathrm{~g} / \mathrm{dl}$. Kidney function tests, muscle enzymes, and urinalysis were normal. Antibiotherapy was initiated after taking blood, throat and urine cultures. Infectious work-up did not reveal any microorganism as shown in Table I. On the 3rd day, she developed hypotension $(80 / 45 \mathrm{mmHg})$, tachycardia, respiratory distress, and oliguria. Laboratory tests showed hypoalbuminemia (albumin $2.1 \mathrm{~g} / \mathrm{dl}$ ), thrombocytopenia (platelets $\left.107,000 / \mathrm{mm}^{3}\right)$, high pro-BNP $(11,030 \mathrm{pg} /$ $\mathrm{ml}$ ) and prolongation of coagulation studies. The clinical picture was thought as toxic shock syndrome and patient was transferred to PICU. On echocardiography, there were no any coronary abnormalities but EF was found to be $55 \%$. Fluid resuscitation, broadspectrum antibiotics and inotropic treatment were initiated. On the 5 th day, pulmonary edema and bilateral pleural effusion developed, and as respiratory distress worsened, noninvasive mechanical ventilation was applied. Albumin, FFP and IVIG ( $1 \mathrm{~g} / \mathrm{kg})$ infusions were given as supportive care. On the 6 th day, she developed bilateral conjunctivitis, rash on perineum, red and cracked lips. The child still had fever with 4-5 peaks per day around 39.5 ${ }^{\circ} \mathrm{C}$. As fever persisted and 48 hours passed after the first IVIG infusion, a second, high dose IVIG $(2 \mathrm{~g} / \mathrm{kg})$ was given by 12 hours' infusion and acetylsalicylic acid was started with the diagnosis of KD. She became afebrile and respiratory symptoms improved after the 2nd dose of IVIG. As the throat culture, anti-streptolysin $\mathrm{O}$ and anti-DNase $\mathrm{B}$ were normal, streptococcal infection was discarded.

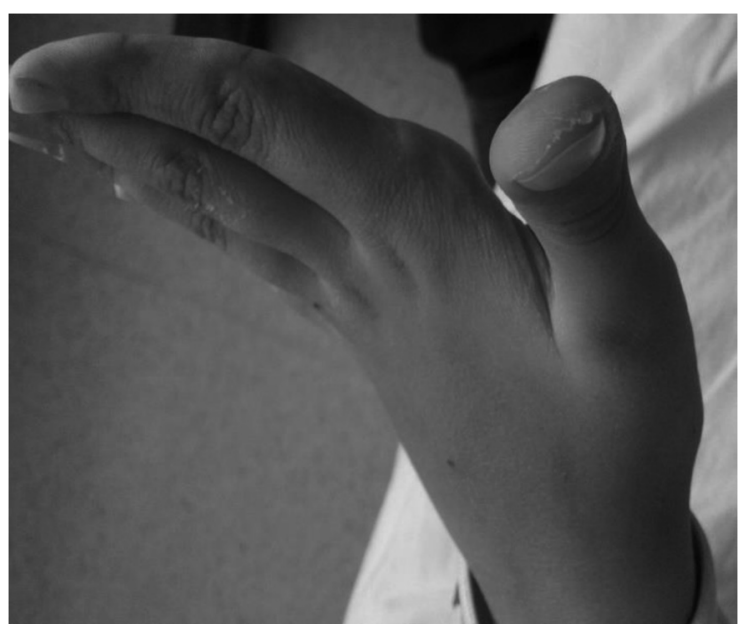

Fig. 1. Periungual desquamation of the thumb 
Table I. Infectious Work-Up of the Cases

\begin{tabular}{lcc}
\hline Parameter & Case 1 & Case 2 \\
\hline Blood culture & Sterile & Sterile \\
Urine culture & Sterile & Sterile \\
Throat culture & Not done & Normal flora \\
Chest X-ray & Bilateral pleural effusion and & Bilateral pleural effusion and \\
Cytomegalovirus IgM & cardiomegaly & Negative \\
Epstein-Barr virus viral capsid & Negative & Negative \\
antigen IgM & Negative & Negative \\
Rubella IgM & Negative & Negative \\
Toxoplasma gondii IgM & Negative & Negative \\
Parvovirus B19 IgM & Negative & Negative \\
Mycoplasma pneumonia IgM & & Negative \\
\hline
\end{tabular}

She developed typical thrombocytosis (platelet: $836,000 / \mathrm{mm}^{3}$ ) and periungual desquamation of KD (Fig. 1) towards the end of the second week and echocardiographic examination was normal by all parameters. The patient is being followed for 9 months without any complication.

\section{Discussion}

Kawasaki disease is a systemic vasculitis of unknown cause and the leading cause of acquired heart disease in the developed countries ${ }^{4}$. First cases of KD was described by Tomisaku Kawasaki in 1962 at the Japanese Pediatric Association meeting in Chiba, Japan, under the name of 'non-scarlet fever syndrome with desquamation'. T. Kawasaki described 50 more cases in Japanese literature in 1967 and for the first time in English literature in 1974 under the name of 'acute mucocutaneous lymph node syndrome' later will be known as $\mathrm{KD}^{5}$. For many years, it is well known that during acute stage of the disease any part of the heart including endocardium, myocardium, pericardium, valves, conduction system, and coronary arteries may be involved ${ }^{1,2,4}$. Myocarditis, tachycardia, pericarditis, valvulitis and conduction deficits can be observed during acute stage but shock is not one of the common forms of presentation of the disease ${ }^{1,2,4,6}$.

KDSS was recently described by Kanegaye et al. ${ }^{3}$ in 2009 and defined as the presence of any of the following conditions: systolic hypotension for age, a sustained decrease in systolic blood pressure from baseline of $\geq 20 \%$ or clinical signs of poor perfusion (tachycardia, prolonged capillary filling time, cool extremities, diminished pulses, oliguria, or mental status changes not accounted for by other conditions such as fever or ambient temperature) with accompanying features of KD. Our two cases had sustained hypotension and other signs of poor perfusion like oliguria, cold extremities and mental status changes. In addition to diagnostic criteria, KD patients may have many nonspecific clinical features like irritability, vomiting, diarrhea, cough, weakness, abdominal pain, arthralgia and myalgia ${ }^{1,2,7}$. Baker et al. ${ }^{7}$ found irritability in $50 \%$ and weakness in $19 \%$ of $198 \mathrm{KD}$ patients and they speculated that these nonspecific symptoms may reflect diffuse vasculitis or be the sequelae of an infectious trigger(s) of KD. Myalgia and fatigue are considered as features that suggest a vasculitic syndrome. ${ }^{8}$ Our two cases had overt weakness, and myalgia that resolved rapidly with the resolution of vasculitis by appropriate treatment. In the literature search, we did not find that patients with intense myalgia or weakness have increased risk for $\operatorname{KDSS}^{3,6,9,10}$.

The cause of KDSS is unknown but capillary leakage due to vasculitis, myocardial dysfunction, and cytokine dysregulation are thought to be responsible,6,9. Kanegaye et al. $^{3}$ found KDSS in $13(7 \%)$ of $187 \mathrm{KD}$ patients and in the study of Gámez-González et al., 10 of 214 consecutive patients with $\mathrm{KD}, 11$ (5\%) met the definition for KDSS.

As clinical pictures are very similar, KDSS can be misdiagnosed as toxic shock syndrome (TSS) or septic shock. İşüder et al. ${ }^{11}$ presented a 
6-year-old girl and Saha et al. ${ }^{12}$ presented a 13-year-old girl with KDSS that both were initially diagnosed as TSS. The case presented by İşüder et al. ${ }^{11}$ was very similar to our second case. She had prolonged fever, tachycardia, hypotension, delayed capillary filling time and mild hypertransaminasemia that had provisional diagnosis of TSS. The case of Thabet et al. ${ }^{13}$ was a 5-month-old girl with KDSS that was initially diagnosed as septic shock. Our first case had the initial diagnosis as KD but the second one was believed to have TSS. The similar presentations of KDSS and TSS (caused by $S$. aureus or $S$. pyogenes) suggest that bacterial superantigens may be involved in the pathogenesis ${ }^{14}$.

In the study of Kanegaye et al. ${ }^{3}$ patients with KDSS had larger proportion of immature neutrophils, higher CRP concentrations, lower hemoglobin concentrations and platelet counts. Evidence of consumptive coagulopathy, impaired left ventricular systolic function, coronary artery abnormalities and IVIG resistance were common in the KDSS group. Gámez-González et al. ${ }^{10}$ showed that patients with KDSS seem to have an increase in gastrointestinal manifestations, incomplete presentation, IVIG resistance, and higher incidence of giant coronary aneurysms and myocardial infarction. Both of our patients had consumptive coagulopathy and hypoalbuminemia requiring FFP and albumin infusions, had very high CRP and ESR levels reflecting intense inflammation. The second case had a more complicated course with thrombocytopenia. Both of the cases had impaired left ventricular systolic function reflected by low ejection fraction and very high pro-BNP levels and although the first case had IVIG resistance and coronary artery ectasia during acute stage, none of them had coronary abnormalities during convalescent phase and follow-up. As in our cases, KDSS patients generally require PICU admission. Apart from acetylsalicylic acid and IVIG, intense supportive treatment including fluid resuscitation, inotropic treatment, and respiratory support are commonly required $3,6,9$.

In conclusion, in patients presenting with fever and shock-like state, KD should be in differential diagnosis and patients should be looked for signs of KD. And in case of clinical doubt, serial echocardiographic examination should be performed to look for coronary involvement.

\section{REFERENCES}

1. Sundel RP, Petty RE. Kawasaki disease. In: Cassidy JT, Petty RE, Laxer ML, Lindsey CB (eds). Textbook of Pediatric Rheumatology. 6 th ed. Philadelphia: Elsevier; 2011: 505-520.

2. Newburger JW, Takahashi M, Gerber MA, et al. Committee on Rheumatic Fever, Endocarditis and Kawasaki Disease, Council on Cardiovascular Disease in the Young, American Heart Association, American Academy of Pediatrics. Diagnosis, treatment, and longterm management of Kawasaki disease: a statement for health professionals from the Committee on Rheumatic Fever, Endocarditis and Kawasaki Disease, Council on Cardiovascular Disease in the Young, American Heart Association. Circulation 2004; 110: 2747-2771.

3. Kanegaye JT, Wilder MS, Molkara D, et al. Recognition of a Kawasaki Disease Shock Syndrome. Pediatrics 2009; 123: e783-e789.

4. Scuccimarri R. Kawasaki disease. Pediatr Clin North Am 2012; 59: 425-445.

5. Burns JC, Kushner HI, Bastian JF, et al. Kawasaki disease: A brief history. Pediatrics 2000; 106: E27.

6. Chen PS, Chi H, Huang FY, Peng CC, Chen MR, Chiu NC. Clinical manifestations of Kawasaki disease shock syndrome: a case-control study. J Microbiol Immunol Infect 2015; 48: 43-50.

7. Baker AL, Lu M, Minich LL, Atz AM, et al. Associated symptoms in the ten days prior to diagnosis of Kawasaki disease. J Pediatr 2009; 154: 592-595.

8. Cassidy JT, Petty RE. Vasculitis and its classification. In: Cassidy JT, Petty RE, Laxer ML, Lindsey CB (eds). Textbook of Pediatric Rheumatology (6 $6^{\text {th }}$ ed). Philadelphia: Elsevier, 2011: 479-482.

9. Gatterre P, Oualha M, Dupic L, et al. Kawasaki disease: an unexpected etiology of shock and multiple organ dysfunction syndrome. Intensive Care Med 2012; 38: 872- 878 .

10. Gámez-González LB, Murata C, Muñoz-Ramírez M, Yamazaki-Nakashimada M. Clinical manifestations associated with Kawasaki disease shock syndrome in Mexican children. Eur J Pediatr 2013; 172: 337-342.

11. İşgüder R, Doksöz Ö, Bağ Ö, et al. Kawasaki disease shock syndrome: a severe form of Kawasaki disease. Turk J Pediatr 2013; 55: 319-321.

12. Saha A, Khalil S, Kapoor K, Gupta A, Dubey NK. Kawasaki shock syndrome presenting as toxic shock syndrome. Int J Rheum Dis 2013; 16: 480-482.

13. Thabet F, Bafaqih H, Al-Mohaimeed S, Al-Hilali M, AlSewairi W, Chehab M. Shock: an unusual presentation of Kawasaki disease. Eur J Pediatr 2011; 170: 941-943.

14. Matsubara K, Fukaya T. The role of superantigens of group A Streptococcus and Staphylococcus auresu in Kawasaki disease. Curr Opin Infect Dis 2007; 20: 298-303. 